\title{
EDITORIAL
}

\section{The role of cytogenetics in myeloma}

Leukemia (2006) 20, 1484-1486. doi:10.1038/sj.leu.2404330

Multiple myeloma is a slowly proliferating disease of 'mature B cells', involving mainly the bone marrow. Active myeloma is often preceded by an indolent phase of monoclonal gammopathy of undetermined significance or smoldering myeloma. Although patients at this stage do not require therapy, genetically, the plasma cells are already very abnormal with the large majority of such patients having an aneuploid DNA content and abnormal cytogenetics by fluorescence in situ hybridization (FISH), including deletion of chromosome 13, $t(11 ; 14), t(4 ; 14), t(6 ; 14), t(14 ; 16)$ and $t(14 ; 20)$, although the latter three translocations are uncommon. ${ }^{1-5}$ In patients with active myeloma, more than $90 \%$ have abnormal cytogenetics by FISH if tested for hyperdiploidy and for the common translocations involving $14 \mathrm{q} 32 .{ }^{6}$ In contrast, using metaphase cytogenetics, only one-third of patients will show an abnormal karyotype, which is usually complex and on an average 11 chromosomes are involved. ${ }^{7}$ The other two-thirds will have normal metaphase cytogenetics. These normal metaphases are not derived from the myeloma cells, but from the remaining normal hematopoietic cells. The reason for failure to have informative mitoses is the low proliferative capacity of the myeloma cells. It is the failure to obtain informative cytogenetics in the majority of patients that has led to a shift away from metaphase cytogenetics to interphase FISH. Deletion of chromosome 13 and hypodiploidy by metaphase cytogenetics are associated with a poor outcome. ${ }^{8-10}$ Based on these findings, it has been assumed that deletion of chromosome 13 by FISH would have a similar poor prognosis as the metaphase abnormality. In fact, in the IFM 99 study, poor prognosis was defined as a combination of deletion 13 by FISH and elevated B2 microglobulin level. ${ }^{11}$ Unfortunately, mixing results of metaphase cytogenetics with those obtained by FISH has resulted in confusion and statements that deletion of chromo- some 13 (as determined by FISH) was not a poor prognostic factor. Patients with inferior outcome as analyzed by FISH were those with $\mathrm{t}(4 ; 14), \mathrm{t}(14 ; 16)$ and gene deletion of $\mathrm{p} 53 .{ }^{12,13}$ Also, it was claimed that on metaphase cytogenetics not deletion 13, but hypodiploidy was associated with poor outcome. ${ }^{9}$ It is clear that often there is an association between hypodiploidy and deletion of chromosome $13 .{ }^{9}$ However, our work has shown that both hypodiploidy without deletion of chromosome 13 and deletion of chromosome 13 without hypodiploidy are associated with a poor prognosis. ${ }^{10}$ When analyzing outcome of patients with or without FISH deletion 13, those with deletion 13 (approximately $50 \%$ of all patients) as a group have an inferior outcome (Figure 1), but if we divide patients with deletion 13 by FISH into those with normal versus abnormal metaphase karyotypes, it becomes evident that the inferior prognosis of patients with FISH deletion 13 is entirely due to the one-third of those patients $(17 \%$ of the overall patient population) with abnormal metaphase cytogenetics, whereas those with normal metaphase cytogenetics and deletion 13 by $\mathrm{FISH}$ have an outcome similar to those without FISH deletion 13 (Figure 2).

In the context of the ongoing confusion of the prognostic value of metaphase versus FISH cytogenetics, the paper in this issue by Laura Chiecchio et al. is very relevant. The authors compared results obtained by $\mathrm{FISH}$, not only for deletion of chromosome 13, but also for hyperdiploidy, p53, $t(14 ; 16)$, $t(14 ; 20), t(6 ; 14)$ and $t(4 ; 14)$, with those obtained by metaphase cytogenetics. This was a large multicenter study including 794 patients, with $78 \%$ of these patients studied at the time of diagnosis. A weakness of the study is that the treatment approach was not uniform and that it is unknown whether the knowledge of cytogenetic results had an impact on the type of therapy given to patients. In addition, the median follow-up was a short 22 months. Their findings confirm our data that abnormal metaphase cytogenetics are associated with a poor outcome and that patients with FISH deletion 13 but without abnormal metaphase cytogenetics had similar outcomes to those with no
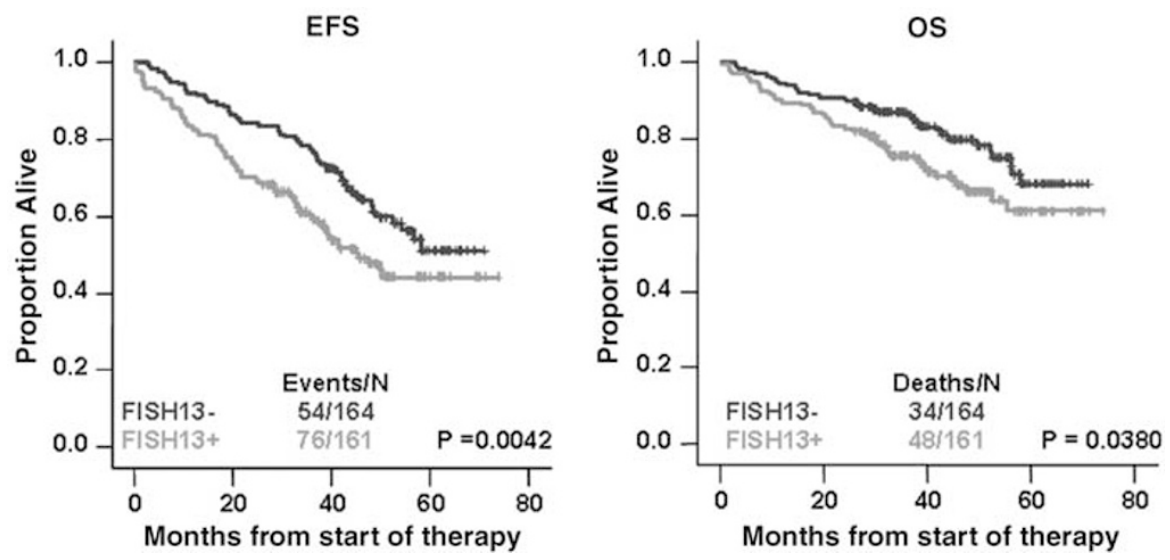

Figure 1 Depicts event-free (EFS) and overall survival (OS) of newly diagnosed patients, treated uniformly on our Total Therapy 2 protocol (with the exception of randomization upfront to thalidomide). Patients with deletion of chromosome 13 detected by FISH had an inferior EFS and OS $(N=161)$. 

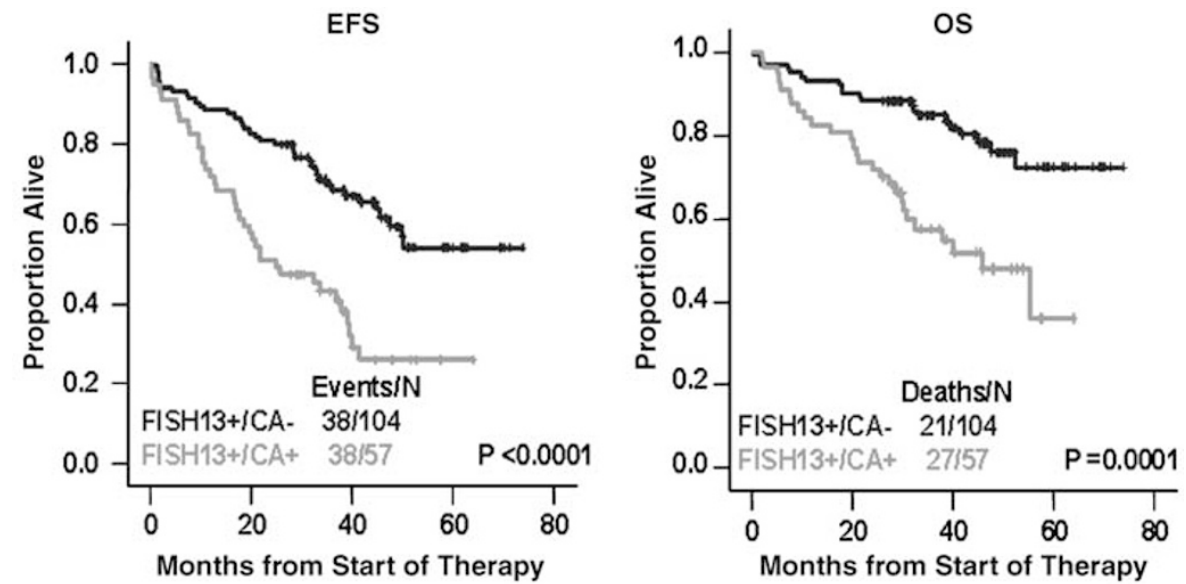

Figure 2 When the group of patients with deletion of chromosome 13 detected by FISH $(N=161)$ were subdivided into those with metaphase cytogenetic abnormalities $(N=57)$ and those without metaphase cytogenetic abnormalities $(N=104)$, the inferior outcome of patients with deletion of chromosome 13 detected by FISH was entirely due to those patients who also had metaphase cytogenetic abnormalities, whereas those with FISH deletion 13, but normal metaphase cytogenetics had EFS and OS similar to patients without FISH deletion 13 (see Figure 1).

FISH deletion 13. Addition of ploidy status to deletion 13 by FISH did not make a difference in survival prediction. Deletion of chromosome 13 by FISH was much more frequent in patients with non-hyperdiploidy, $\mathrm{t}(4 ; 14)$ and deletion of $\mathrm{p} 53$. The new and interesting findings of this paper relate to the fact that patients with 'poor prognostic' FISH markers such as $\mathrm{t}(4 ; 14)$ or deletion p53 without abnormal metaphase cytogenetics had outcomes comparable to those without these poor prognostic markers and those with normal metaphase cytogenetics. A poor outcome was only seen in those with the poor FISH markers, who also had abnormal metaphase cytogenetics, which is reminiscent of what happens to patients with FISH deletion 13. Some of our influential colleagues consider the prognosis of patients with $\mathrm{t}(4 ; 14)$ so grim even with tandem transplants, that they do not recommend those patients to proceed with transplantation. ${ }^{14}$ Based on the data published in this paper, those with $t(4 ; 14)$ but normal metaphase cytogenetics have a 3 -year survival that is not significantly different from those without $t(4 ; 14)$. It should be remembered that the median follow-up in this study was only 22 months and, therefore, the relatively stable part of the Kaplan-Meier curves is the initial part. Our own data show a 5-year event-free and overall survival of all patients with this translocation of 15 and $51 \%$, respectively (Figure 3 ). Therefore, the $t(4 ; 14)$ entity is not a homogeneous group, with subgroups of patients with such a translocation faring better than others. Thus, denial of transplantation for those patients is not justified.

Why are the results of metaphase cytogenetics so much more informative than FISH cytogenetics? Metaphase cytogenetic analysis is a biological test. If myeloma cells are stromadependent, as is the case in early myeloma, taking these myeloma cells out of their supportive microenvironment will result in apoptosis of myeloma cells, and therefore no informative mitoses. If myeloma cells have become stromaindependent in the advanced stages of the disease, taking the myeloma cell out of the microenvironment will not result in their cell death and the myeloma cells will not only survive, they will proliferate and give rise to abnormal mitoses. Finding abnormal mitoses is still the best surrogate marker we have available at this time for stroma-independent and therefore advanced myeloma. ${ }^{15}$ Just like in acute leukemia, it is critical that all new myeloma patients have a sample analyzed for metaphase cytogenetics. There is no problem with performing

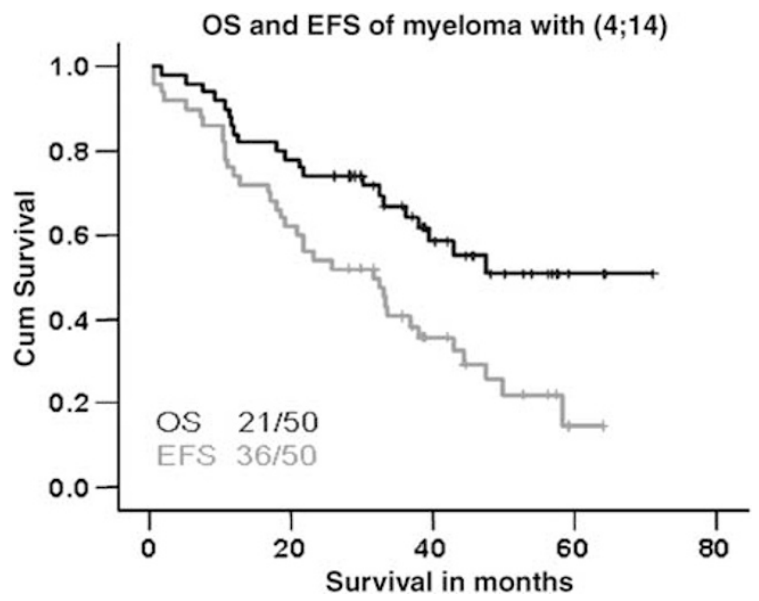

Figure 3 Although the EFS of patients with $t(4 ; 14)$ as determined by gene expression profiling is poor with less than $20 \%$ event-free at 5 years, more than $80 \%$ of patients with this translocation were still alive at 5 years after enrollment in a tandem transplantation protocol.

additional FISH cytogenetics, but it should not replace metaphase cytogenetics because it has an inferior prognostic significance.

F Zhan ${ }^{1}$, J Sawyer ${ }^{2}$ and G Tricot $^{1}$

${ }^{1}$ Myeloma Institute for Research and Therapy, University of Arkansas for Medical Sciences, Little Rock, AR, USA and ${ }^{2}$ Department of Pathology, University of Arkansas for Medical Sciences, Little Rock, AR, USA E-mail: tricotguidoj@uams.edu

\section{References}

1 Drach J, Angerler J, Schuster J, Rothermundt C, Thalhammer R, Haas $\mathrm{O}$ et al. Interphase fluorescence in situ hybridization identifies chromosomal abonormalities in plasma cell from patients with monoclonal gammopathy of undetermined significance. Blood 1995; 86: 3915-3921.

2 Avet-Loiseau H, Facon T, Grosbios B, Magrangeas F, Rapp MJ, Harousseau JL et al. Oncogenesis of multiple myeloma: $14 q 32$ and $13 q$ chromosomal abnormalities are not randomly distributed, but 
correlate with natural history, immunological features, and clinical presentation. Blood 2002; 99: 2185-2191.

3 Fonseca R, Bailey R, Ahmann G, Rajkumar SV, Hoyer JD, Lust JA et al. Genomic abnormalities in monoclonal gammopathy of underdetermined significance. Blood 2002; 100: 1417-1424.

4 Shaughnessy J, Gabrea A, Qi Y, Brents L, Zhan F, Tian E et al. Cyclin D3 at 6p21 is dysregulated by recurrent chromosomal translocations to immunoglobulin loci in multiple myeloma. Blood 2001; 98: 217-223.

5 Boersma-Vreugdenhil GR, Kuipers J, Van Stralen E, Peeters T, Michaux L, Hagemeijer A et al. The recurrent translocation $t(14 ; 20)(q 32 ; q 12)$ in multiple myeloma results in aberrant expression of MAFB: a molecular and genetic analysis of the chromosomal breakpoint. Br J Haematol 2004; 126: 355-363.

6 Zandecki M, Lai JL, Facon T. Multiple myeloma: almost all patients are cytogenetically abnormal. Br J Haematol 1996; 94: 217-227.

7 Tricot G, Sawyer J, Jagannath S, Desikan R, Siegel D, Naucke S et al. Unique role of cytogenetics in the prognosis of patients with myeloma receiving high-dose therapy and autotransplants. J Clin Oncol 1997; 15: 2659-2666.

8 Tricot G, Barlogie B, Jagannath S, Bracy D, Mattox S, Vesole D et al. Poor prognosis in multiple myeloma is associated only with partial or complete deletions of chromosome 13 or abnormalities involving $11 \mathrm{q}$ and not with other karyotype abnormalities. Blood 1995; 86: 4250-4256.
9 Smadja NV, Bastard C, Brigaudeau C, Leroux D, Fruchart C. Hypodiploidy is a major prognostic factor in multiple myeloma. Blood 2001; 98: 2229-2238.

10 Fassas A, Spencer T, Sawyer J, Zangari M, Lee CK, Anaissie E. Both hypodioploidy and deletion of chromosome 13 independently confer poor prognosis in multiple myeloma. Br J Haematol 2002; 118: 1041-1047.

11 Facon T, Avet-Loiseau H, Guillerm G, Moreau P, Genevieve F, Zandecki $\mathrm{M}$ et al. Chromosome 13 abornmalities identified by FISH analysis and serum $\beta 2$-microglobulin produce a powerful myeloma staging system for patients receiving high-dose therapy. Blood 2001; 97: 1566-1571.

12 Stewart AK, Fonseca R. Prognostic and therapeutic significance of myeloma genetics and gene expression profiling. J Clin Oncol 2005; 23: 6339-6344.

13 Dewald G, Therneau T, Larson D, Lee YK, Fink S, Smoley S et al. Relationship of patient survival and chromosome anomalies detected in metaphase and/or interphase cells at diagnosis of myeloma. Blood 2005; 106: 3553-3558.

14 Chang H, Sloan S, Li D, Zhuang L, Yi QL, Chen C et al. The t( $4 ; 14)$ is associated with poor prognosis in myeloma patients undergoing autologous stem cell transplant. Br J Haematol 2004; 125: 64-68.

15 Tricot G, Barlogie B, van Rhee F. Treatment advance in multiple myeloma. Br J Haematol 2004; 125: 24-30. 